\title{
High-Brightness Injection-Seeded Soft-X-Ray-Laser Amplifier Using a Solid Target
}

\author{
Y. Wang, E. Granados, M. A. Larotonda, M. Berrill, B. M. Luther, D. Patel, C. S. Menoni, and J. J. Rocca \\ NSF ERC for Extreme Ultraviolet Science and Technology and Department of Electrical and Computer Engineering, \\ Colorado State University, Fort Collins, Colorado 80523, USA
}

(Received 15 July 2006; published 18 September 2006)

\begin{abstract}
We demonstrate the generation of an intense soft-x-ray-laser beam by saturated amplification of high harmonic seed pulses in a dense transient collisional soft-x-ray-laser plasma amplifier created by heating a titanium target. Amplification in the $32.6 \mathrm{~nm}$ line of Ne-like Ti generates laser pulses of subpicosecond duration that are measured to approach full spatial coherence. The peak spectral brightness is estimated to be $\sim 2 \times 10^{26}$ photons $/\left(\mathrm{s} \mathrm{mm}^{2} \mathrm{mrad}^{2} 0.01 \%\right.$ bandwidth). The scheme is scalable to produce extremely bright lasers at very short wavelengths with full temporal and spatial coherence.
\end{abstract}

There is great interest in the generation of highbrightness beams of soft-x-ray radiation. The emerging fourth generation synchrotron sources designed to provide highly coherent soft-x-ray beams of greatly increased brightness will open new opportunities in science [1]. However, the widespread use of intense soft-x-ray light requires the development of significantly more compact and less expensive coherent sources. With this objective, significant efforts are presently focused in the development of tabletop sources based on either the amplification of spontaneous emission in a plasma [2] or the nonlinear upconversion of optical laser light into soft-x-ray light [3]. The first of these processes is a promising route for the generation of high energy soft-x-ray-laser pulses of high monochromaticity using tabletop devices. The achievement of high brightness requires the demonstration of high spatial coherence. At a wavelength of $46.9 \mathrm{~nm}$, intense laser beams with essentially full spatial coherence have been produced in capillary discharge plasmas [4]. However, the self-amplification of spontaneous emission in the much denser laser-created plasmas required to generate soft-x-ray-laser beams at shorter wavelengths generates beams of limited spatial coherence [5]. To overcome this limitation, seeding of the soft-x-ray-laser amplifier with either the spatially filtered radiation from a second soft-x-ray amplifier [6,7] or with a high harmonic of an optical laser has been investigated [8-10]. The latter scheme has the advantage of producing significantly shorter (subpicosecond) laser pulses and also requires less laser pump energy to generate the seed pulse. Saturated amplification of the 25th harmonic of a Ti:sapphire (Ti:Sa) laser was recently demonstrated in a $32.8 \mathrm{~nm}$ Ni-like $\mathrm{Kr}$ optical field ionization (OFI) soft$\mathrm{x}$-ray-laser amplifier [9]. However, the comparatively low plasma density $\left(<1 \times 10^{19} \mathrm{~cm}^{-3}\right)$ at which optimum lasing occurs in these OFI lasers results in a relatively low saturation intensity and narrow bandwidth that will ultimately limit the maximum brightness possible using that scheme. Therefore, there is much interest in the demonstration of high harmonic seeding of collisional soft- $x$-ray- laser amplifiers based on the dense plasmas created by laser heating of solid targets, which have been demonstrated to operate in the gain saturation regime at wavelengths as short at $5.9 \mathrm{~nm}$ [11]. The up to 2 orders of magnitude larger electron density in this type of plasma amplifier results in a significantly increased saturation intensity and a broader laser linewidth, opening a route that can lead to higher laser pulse intensities and shorter pulse widths. An early experiment performed in a Ne-like Ga plasma amplifier pumped by overlapping three $200 \mathrm{~J}$ optical laser beams onto a solid gallium target demonstrated the amplification of the harmonic seed, but only by a factor of about 3 [7]. More recently, a Ne-like Mn soft$\mathrm{x}$-ray-laser amplifier was reported to amplify a high harmonics seed from $4.7 \mathrm{pJ}$ to $3 \mathrm{~nJ}$ [10].

Herein, we report several significant advances in highbrightness tabletop soft-x-ray-laser development. First, we report what to our knowledge is the first demonstration of saturated amplification of a high harmonic seed in the high density plasma of a transient collisional soft-x-ray laser created by heating a solid target. A $32.6 \mathrm{~nm}$ tabletop soft-xray-laser amplifier operating in the $3 p^{1} S_{0} \rightarrow 3 s^{1} P_{1}$ line of $\mathrm{Ne}$-like Ti at $5 \mathrm{~Hz}$ repetition rate [12] was used to amplify a seed pulse from the 25th harmonic of Ti:Sa into the gain saturation regime. The result is scalable to produce extremely bright lasers at very short wavelengths. Second, we demonstrate that the resulting soft-x-ray beam is essentially fully spatially coherent. Third, we demonstrate that in special cases it is possible to simultaneously seed more than one laser line to produce highly coherent soft-x-raylaser beams at two different wavelengths. The 27th harmonic of Ti:Sa was simultaneously amplified in the $30.1 \mathrm{~nm} 3 d^{1} P_{1} \rightarrow 3 p{ }^{1} P_{1}$ line of Ne-like Ti in the same plasma. Fourth, we discuss the measurements in comparison with modeling results that describe the dynamics of seeded amplification in a dense collisionally pumped soft$\mathrm{x}$-ray-laser amplifier. The model simulations indicate that the soft-x-ray-laser pulses are subpicosecond in duration. Finally, the results were obtained with a tabletop laser operating at a repetition rate of $5 \mathrm{~Hz}$, showing that this is 
a practical scheme to produce extremely high-brightness soft-x-ray beams for applications in a small laboratory environment.

The experimental setup is schematically illustrated in Fig. 1. A single tabletop Ti:Sa laser driver operating at $5 \mathrm{~Hz}$ repetition rate was used to create both the harmonic seed pulse and the soft-x-ray-laser amplifier. It consists of a chirped pulse amplification laser system $(\lambda=800 \mathrm{~nm})$ comprising three stages of amplification. A beam splitter at the output of the second amplification stage separates a $30 \mathrm{~mJ}$ pulse for the generation of the harmonic seed. The rest of the energy is directed to the third amplification stage, where it is amplified to obtain a $\sim 1.5 \mathrm{~J}$ pulse. The soft-x-ray-laser amplifier consists of a line focus plasma of up to $4 \mathrm{~mm}$ in length generated by exciting a $2 \mathrm{~mm}$ thick polished Ti slab target. The plasma is formed by a $0.3 \mathrm{~J}$ prepulse, which arrives $\sim 5 \mathrm{~ns}$ after a $\sim 10 \mathrm{~mJ}$ pulse. The plasma is allowed to expand to reduce the density gradient and is subsequently rapidly heated by a $\sim 800 \mathrm{~mJ}, 6.7 \mathrm{ps}$ duration pulse impinging at a grazing incidence angle of $23^{\circ}$ onto the target for efficient plasma heating [13-16]. The prepulse beam is focused into a $\sim 30 \mu \mathrm{m}$ wide, $4.1 \mathrm{~mm}$ FWHM line focus. The short pulse is focused into a similar line focus utilizing a parabolic mirror of $f=$ $76.2 \mathrm{~cm}$ positioned at $7^{\circ}$ from normal incidence. The target surface is tilted to form the grazing incidence angle of $\theta=23^{\circ}$ with respect to the axis of the short pulse beam. At this angle of incidence, $\theta=\left(n_{e} / n_{c}\right)^{1 / 2}$ refraction couples the pump beam energy into the plasma region where the electron density is $n_{e}=2.6 \times 10^{20} \mathrm{~cm}^{-3}$. High harmonic pulses of a few nanojoules energy are generated using $20 \mathrm{~mJ}$ drive pulses compressed to $\sim 50 \mathrm{fs}$. These pulses are focused by a $f=5 \mathrm{~m}$ lens into an $8.8 \mathrm{~cm}$ long gas cell filled with 5 Torr of argon. The center wavelength of the 25th harmonic is matched to the $32.6 \mathrm{~nm}$ wavelength of the highest gain laser line in the Ti amplifier by adjusting the compressor. This broadens the drive pulse duration to about $130 \mathrm{fs}$, reducing the energy of the seed pulse to 0.5 $1 \mathrm{~nJ}$. The harmonic seed beam exiting the gas cell is relay imaged onto a $\sim 100 \mu \mathrm{m}$ diameter spot at the entrance of the soft-x-ray plasma amplifier using a gold-coated toroidal mirror placed at a grazing angle of $9^{\circ}$. A delay stage is

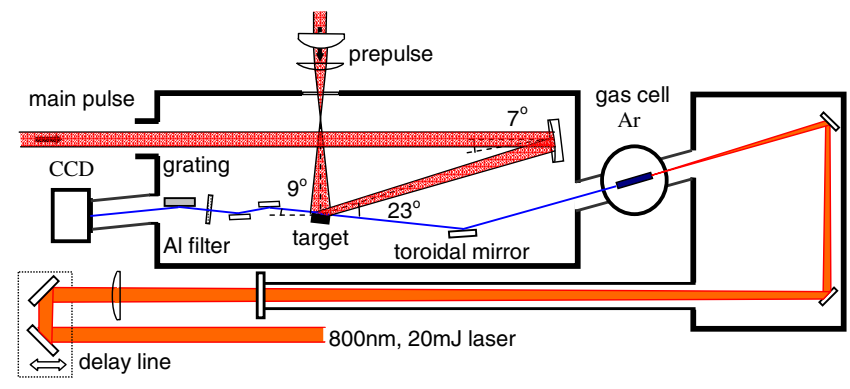

FIG. 1 (color online). Schematic representation of the seeded soft-x-ray-laser amplifier based on a grazing incidence pumped plasma. placed before the $5 \mathrm{~m}$ focusing lens to adjust the delay time between the harmonic pulses and the main pump pulse. The output of the soft-x-ray amplifier is analyzed using a flat field variable spaced 1200 lines $/ \mathrm{mm}$ gold-coated spherical grating set at $3^{\circ}$ grazing incidence and a backilluminated CCD detector placed $48 \mathrm{~cm}$ from the target. Two grazing incidence BK7 flats are used to separate the soft-x-ray laser from the Ti:Sa beam. Spectral filtering and attenuation is achieved using an $\mathrm{Al}$ filter.

Figure 2 illustrates the dramatic increase in the output of a $3 \mathrm{~mm}$ long, $32.6 \mathrm{~nm} \mathrm{Ne}$-like Ti amplifier and the large decrease in the beam divergence achieved by seeding the amplifier. Figure 2(a) shows the spectra of the unseeded Ti soft-x-ray-laser amplifier and the corresponding intensity distribution in the direction parallel to the target. The laser line at $32.6 \mathrm{~nm}$ dominates the spectra and has a divergence of about $10 \mathrm{mrad}$. The detection system can resolve the harmonic seed but not the laser linewidth of $\Delta \lambda / \lambda<1 \times$ $10^{-4}$. Figure 2(b) shows the much lower divergence, about $1 \mathrm{mrad}$, but significantly broader spectra of the harmonic seed. The seeded amplifier output shown in Fig. 2(c) consists of a highly monochromatic spectral line with an energy that is $\sim 64$ times larger that of the seed pulse. The FWHM beam divergence is observed to be about $2.2 \mathrm{mrad}$, considerably smaller than that of the unseeded laser. Figure 3 shows the variation of the intensity of the amplified seed pulse as a function of delay between the 6.7 ps pump pulse and the arrival of the harmonic seed pulse. The time span during which the seed pulse is amplified is determined by the duration of the gain in the

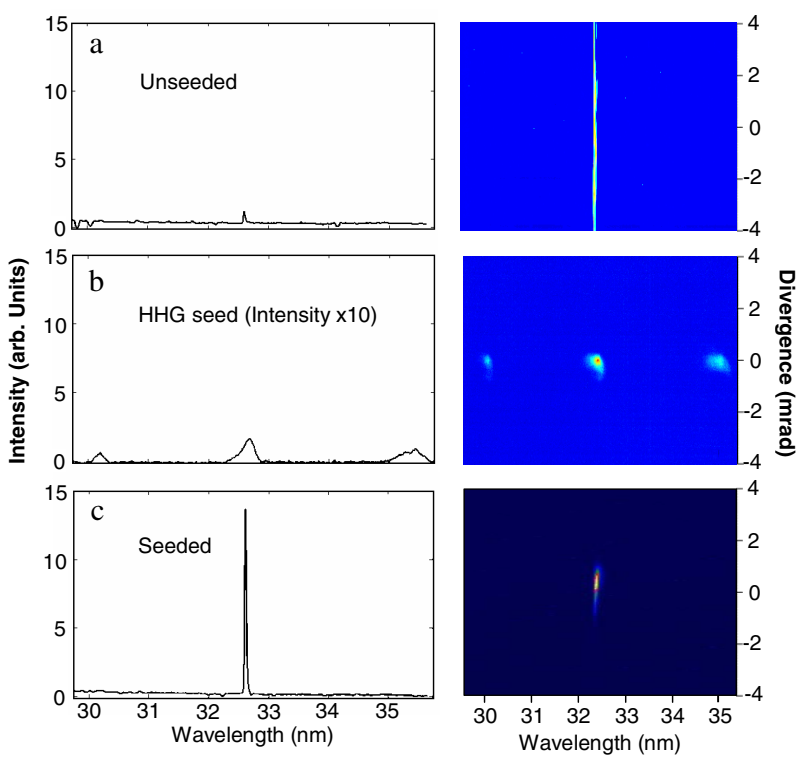

FIG. 2 (color online). Spectra illustrating the relative intensity and beam divergence for the (a) unseeded $32.6 \mathrm{~nm}$ soft-x-raylaser amplifier, (b) high harmonic seed pulse, and (c) seeded soft-x-ray-laser amplifier. The length of the plasma amplifier is $3 \mathrm{~mm}$. The intensity scale of the seed pulse is magnified by 10 times. 




FIG. 3. Variation of the intensity of the amplified seed pulse as a function of time delay between the peak of the 6.7 ps laser amplifier pump pulse and the arrival of the high harmonic seed pulse.

amplifier [17] and is about 5 ps. The maximum amplification is observed at delays between 2 and 4 ps. We also observed that it is possible to simultaneously seed two spectral lines. This is made possible by the fact that the wavelength separation between the 25 th and 27 th harmonics approximately matches the wavelength difference between the 32.6 and $30.1 \mathrm{~nm}$ lines of Ne-like Ti. For the pump conditions used in the experiment, the unseeded output of the $30.1 \mathrm{~nm}$ laser line was observed to be very weak. In contrast, when the wavelength of the harmonics was adjusted to simultaneously obtain sufficient overlap with the two laser lines, intense low divergence output was obtained for both lines, as illustrated in Fig. 4.

Figure 5 illustrates the measured increase of the energy of the $32.6 \mathrm{~nm}$ seed pulse as a function of amplifier length for a time delay of $4 \mathrm{ps}$. The data were obtained by varying the length of the target between 0 and $4 \mathrm{~mm}$ while maintaining both the seed pulse and the amplifier pump excita-

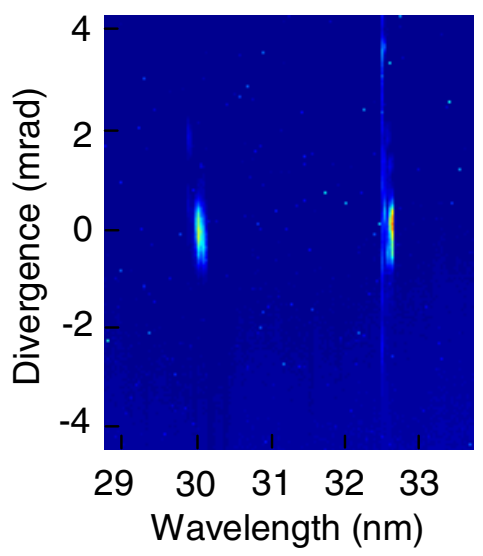

FIG. 4 (color online). Spectra showing the simultaneous seeded amplification in the 32.6 and $30.1 \mathrm{~nm}$ lines of Ne-like Ti. tion conditions constant. The measurements are compared with the results of model simulations in the same figure. The simulations were conducted using a $1 \frac{1}{2}$-dimension hydrodynamic/atomic physics code with multicell radiation transport to compute the evolution of the gain and plasma density profile of the Ne-like Ti amplifier [18]. The propagation and amplification of a $0.7 \mathrm{~nJ}$ seed pulse was computed using a ray-tracing postprocessor code taking into account the effects of gain narrowing and gain saturation. The experimentally measured amplification behavior is very similar to that predicted by the code and can be divided into three distinct phases. The first phase, which takes place in the first $\sim 1 \mathrm{~mm}$ of the amplifier, is dominated by the gain narrowing of the seed pulse which initial $0.1 \mathrm{~nm}$ spectral bandwidth greatly exceeds that of the laser line. This leads to the amplification of only a fraction of its bandwidth, resulting in the observed slow initial seed pulse energy increase. When the seed pulse bandwidth narrows sufficiently to approach the laser linewidth, a second amplification phase starts in which a quasiexponential increase in the energy of the seed pulse takes place. This rapid increase ends after about $2.5 \mathrm{~mm}$ into the amplifier. At this length, the measured amplified seed pulse energy reaches a value consistent with the computed saturation intensity for the $32.6 \mathrm{~nm}$ line of Ne-like $\mathrm{Ti}$ at a plasma density of $2.6 \times 10^{20}$ electrons $/ \mathrm{cm}^{3}$. The third amplification phase corresponds to the gain saturated regime in which efficient energy extraction occurs. The maximum measured amplified seed pulse energy 50-60 nJ is similar to that predicted by the model. The model simulation of the pulse propagation in the amplifier predicts a pulse duration of $0.5-1$ ps determined by the gain-narrowed bandwidth of

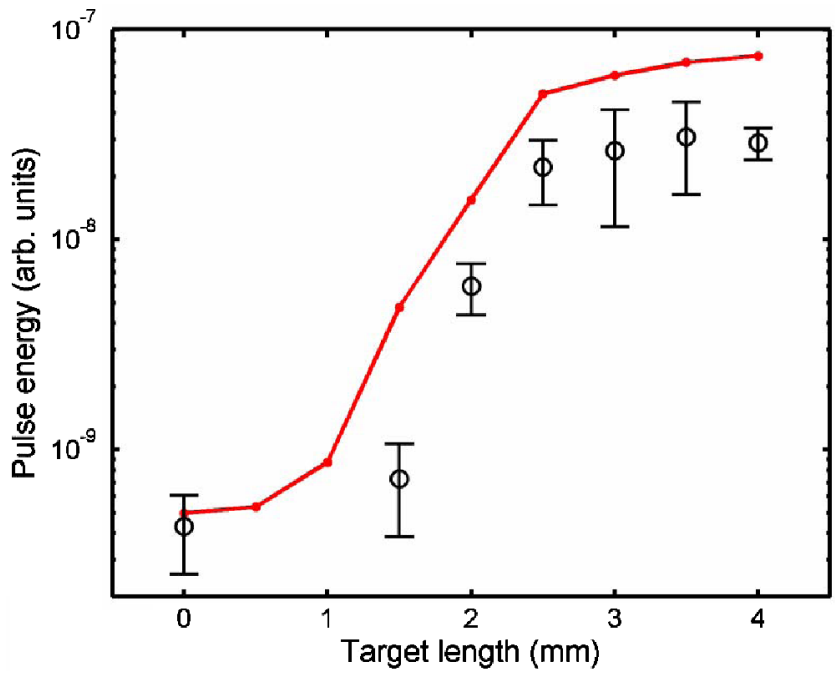

FIG. 5 (color online). Measured and computed (continuous line) variation of the intensity of the amplified seed pulse as a function of plasma amplifier length for the $32.6 \mathrm{~nm}$ line of Nelike Ti. The measured beam reaches the saturation intensity after $\sim 2.5 \mathrm{~mm}$ into the plasma. 


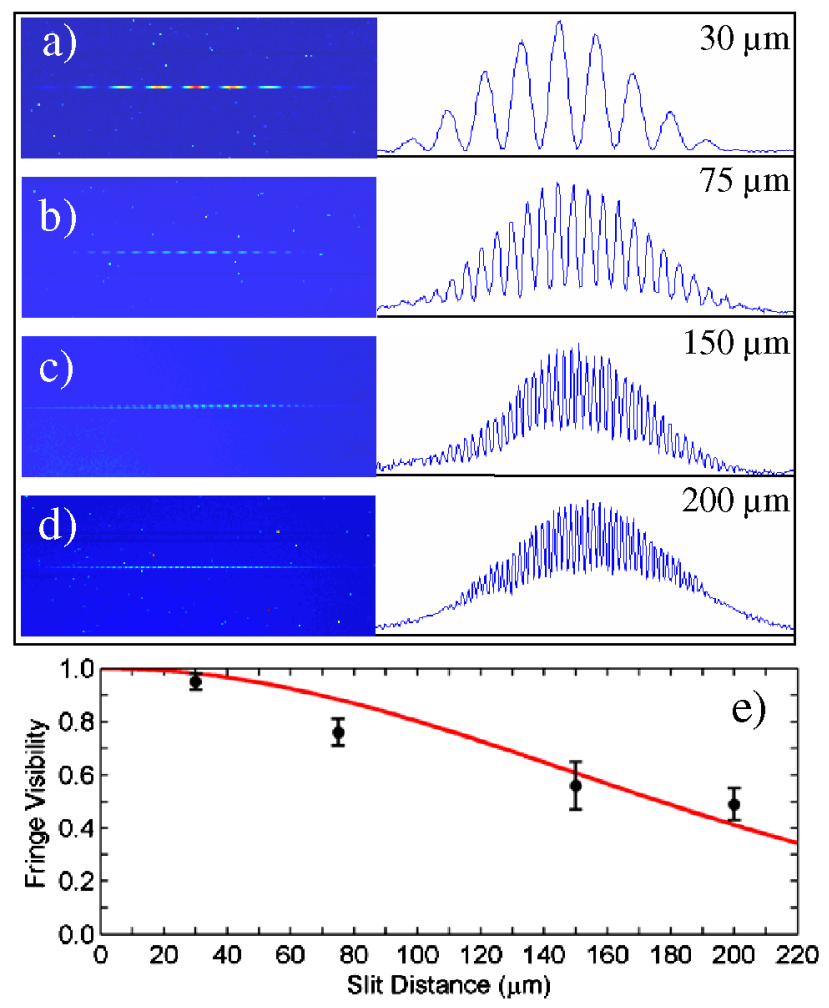

FIG. 6 (color online). Results of Young's slit pair interference experiment for the output of the seeded $32.6 \mathrm{~nm}$ laser amplifier. (a) -(d) Interferograms and their lineout for the slit separation indicated. (e) Plot of the degree of coherence as a function of the slit separation.

the amplified laser transition, which is an order of magnitude shorter than the amplifier gain lifetime.

Measurements in grazing incidence transient collisional lasers have shown that the spatial coherence length is nearly an order of magnitude smaller than the beam diameter [5]. The results of the Young's slit interference experiment illustrated in Fig. 6 show that the degree of spatial coherence improves dramatically when the amplifier is seeded. Pairs of $5 \mu \mathrm{m}$ wide slits separated by 30,75 , 150 , and $200 \mu \mathrm{m}$ were placed at $10 \mathrm{~cm}$ from the exit of the amplifier, a location at which the FWHM beam diameter is about $200 \mu \mathrm{m}$. The measured fringe visibility as a function of slit separation is illustrated in Fig. 6(e). A fit of the data with a Gaussian profile yields a coherent length of $L_{c}=$ $150 \mu \mathrm{m}$. The measurement shows the beam approaches full spatial coherence as the majority of beam energy falls within a coherent length. The equivalent incoherent source diameter of this laser is about $6.9 \mu \mathrm{m}$.

Using the measured beam parameters mentioned above, and assuming a pulse duration of $0.5-1 \mathrm{ps}$ and a horizontal divergence equal to half the vertical divergence, the peak spectral brightness of this source can be estimated to be (1.4-2.8) $\times 10^{26}$ photons $/\left(\mathrm{s} \mathrm{mm}^{2} \mathrm{mrad}^{2} 0.01 \%\right.$ bandwidth $)$. Moreover, it should be noticed that the linewidth of this laser is nearly 20 times narrower than the $0.1 \%$ bandwidth used to specify the brightness of synchrotrons and free electron lasers, an advantage in applications requiring high photon flux in a narrow bandwidth. With excellent temporal and spatial coherence, these lasers are themselves attractive seed sources for mode control in free electron lasers. In conclusion, we have demonstrated a high coherence seeded solid target laser amplifier that is readily scalable to significantly shorter wavelengths and higher plasma densities, leading towards tabletop soft-x-ray sources of extremely high peak brightness.

We thank Henry Kapteyn, David Attwood, Margaret Murnane, and Yanwei Liu for their contributions. This work was supported by the NSF ERC for Extreme Ultraviolet Science and Technology under NSF Grant No. EEC-0310717.

[1] V. Ayvazyan et al., Eur. Phys. J. D 37, 297 (2006).

[2] H. Daido, Rep. Prog. Phys. 65, 1513 (2002); J. J. Rocca, Rev. Sci. Instrum. 70, 3799 (1999).

[3] H. C. Kapteyn et al., Phys. Today 58, No. 3, 39 (2005).

[4] Y. Liu et al., Phys. Rev. A 63, 033802 (2001).

[5] M. A. Larotonda et al., IEEE J. Sel. Top. Quantum Electron. 10, 1363 (2004); Y. Liu et al. (unpublished).

[6] B. Rus et al., Phys. Rev. A 51, 2316 (1995).

[7] M. Nishikino et al., Phys. Rev. A 68, 061802(R) (2003).

[8] T. Ditmire et al., Phys. Rev. A 51, R4337 (1995).

[9] P. Zeitoun et al., Nature (London) 431, 426 (2004).

[10] T. Kawachi et al., Proc. SPIE Int. Soc. Opt. Eng. 5919, 59190L (2005).

[11] R. Smith et al., Phys. Rev. A 59, R47 (1999).

[12] D. Alessi et al., Opt. Express 13, 2093 (2005).

[13] R. Keenan et al., Phys. Rev. Lett. 94, 103901 (2005).

[14] B. M. Luther et al., Opt. Lett. 30, 165 (2005).

[15] Y. Wang et al., Phys. Rev. A 72, 053807 (2005).

[16] J. J. Rocca et al., Opt. Lett. 30, 2581 (2005).

[17] T. Mocek et al., Phys. Rev. Lett. 95, 173902 (2005).

[18] M. Berrill and J. J. Rocca (unpublished). 